\title{
A Novel Approach Using Flexible Scheduling and Aggregation to Optimize Demand Response in the Developing Interactive Grid Market Architecture
}

\author{
Ehsan Reihani, Mahdi Motalleb, Matsu Thornton, Reza Ghorbani ${ }^{1}$
}

\begin{abstract}
-
With the increasing presence of intermittent renewable energy generation sources, variable control over loads and energy storage devices on the grid become even more important to maintain this balance. Increasing renewable energy penetration depends on both technical and economic factors. Distribution system consumers can contribute to grid stability by controlling residential electrical device power consumed by water heaters and battery storage systems. Coupled with dynamic supply pricing strategies, a comprehensive system for demand response (DR) exist. Proper DR management will allow greater integration of renewable energy sources partially replacing energy demand currently met by the combustion of fossil-fuels. An enticing economic framework providing increased value to consumers compensates them for reduced control of devices placed under a DR aggregator. Much work has already been done to develop more effective ways to implement DR control systems. Utilizing an integrated approach that combines consumer requirements into aggregate pools, and provides a dynamic response to market and grid conditions, we have developed a mathematical model that can quantify control parameters for optimum demand response and decide which resources to switch and when. In this model, optimization is achieved as a function of cost savings vs. customer comfort using systematic mathematical market analysis. Two market modeling approaches - the Cournot and SFEare presented and compared. A quadratic function is used for presenting the cost function of each DRA (Demand Response Aggregator) which will be used for settling down the DR market. Contribution of each aggregator and the final price are presented. Finally, we have also performed sensitivity analysis on the house cost function's coefficients for one of the aggregators.
\end{abstract}

Index Terms — Demand response market, demand response scheduling

\section{INTRODUCTION}

In the absence of widespread battery storage systems, thermal energy storage systems, already ubiquitous within the grid, would allow a large degree of control to the supply and demand requirements of the active power grid without requiring further investment. Control over these decentralized thermal energy storage systems, such as domestic water heaters and air conditioning systems, provides grid operators with a powerful tool for the optimization of grid efficiency. Human consumption of thermal energy in the form of hot water may be greater than all other uses combined, typically $40-60 \%$ of total usage in Hawaii. The energy required for water heating is incidentally stored in the hot water tank for later use by the consumer, making these loads an ideal candidate to stagger throughout the day during off-peak times of grid power demand. The effective control and scheduling of these storage devices in the distribution grid gives operators the ability to more effectively harness the inherently variable power generation from distributed renewable energy sources, such as photovoltaic arrays.

In this way, the utility gains these services:

- Load leveling: Controllable loads may be shifted to times of off-peak demand or to make use of available renewable energy generation.

- Grid Stability: Dynamically switching loads helps to stabilize the transient and steady states of the grid.

Improvements in IT infrastructure have provided technologies to communicate with and control grid-tied devices in a cheap and reliable way. The utility system's operator can easily access real-time data about availability of DR resources, and with the application of an algorithm, add or shed loads from the system appropriately to achieve the above specified goals. Demand response can be classified into two general categories: direct load control (DLC) and incentive-based control. In the DLC program, utilities have remote access to switch controllable loads, such as water heaters and air conditioners. A distributed DLC scheme for large-scale residential DR using a two-layer communication-based control architecture has already been proposed [1]. The idea was to allocate the overall control task into each building's energy management controller in a distributed way. A DR program has also been designed in [2] to mitigate peak load during hot summer days using DLC and dynamic price control.

\footnotetext{
${ }^{1}$ Ehsan Reihani, Mahdi Motalleb, Matsu Thornton and Reza Ghorbani are with Renewable Energy Design Laboratory (REDLab) at the Department of Mechanical Engineering, University of Hawaii Manoa, Honolulu, Hawaii, 96822. Phone: +1-808-956-2292, Fax: +1-808-956-0767. E-mail: ereihani@hawaii.edu, $\underline{\text { motalleb@hawaii.edu, matsut@hawaii.edu, rezag@ hawaii.edu }}$
} 
An experimental study was performed in [3] to analyze the effects of DLC-induced temperature cycles on university students' thermal sensation and thermal acceptability in lecture theatres as a worst case setting for DLC-induced thermal environments. The results show that operative temperature, vapor pressure, and the rate of temperature change are the three most important predictors during DLC events. DLC has been implemented successfully in several areas including Hawaii, where Hawaiian Electric has a total of 34,000 DLC customers, collectively providing 15 megawatts of controllable peak demand power. The potential of DRs are studied in [4] in Hawaii in balancing supply and demand on an hourly basis. Much research has been completed concerning various energy market creation strategies employing DR resources [5, 6, 7, 8] . In [9] effects of optimal DR resource reserve scheduling, on the system pollution cost were analyzed. Shen et al. [10] provided an overview of how electricity market policy and regulation reforms have allowed DR to become a viable demand-side resource to address the energy and environmental challenges.

Incentive-based control is implemented by dynamically adjusting the price of energy, ideally as a real-time reflection of the marginal cost of production $[11,12]$. The idea is that given this financial incentive, consumers will adjust their usage [13, 14]. This is sometimes referred to as "time of use pricing", where utilities adjust prices during several periods throughout the day [15, 16, 17]. In its simplest form, this involves only two time steps - peak, and off-peak. With the advent off reliable, cheap, and efficient means of communication and monitoring systems, a far greater resolution may be achieved; especially when coupled with real-time cost analysis. Smaller time steps can be used with pricing that reflects actual real-time marginal cost of production more accurately [18]. Distributed equipment connected to the grid that is capable of participating in demand response can be programmed to process received RTP information and react autonomously based on user defined policies [19, 20]. A model was developed in [21] for optimal behavior of plug-in electric vehicle parking lots in the energy and reserve markets. This model included optimal strategies of parking lots, as responsive demands, in both price-based and incentive-based DR programs. In a similar research [22], a multi-stage stochastic model of a plug-in electric vehicle aggregation agent was proposed to participate in day-ahead and intraday electricity markets. A new trading framework was proposed in [23] which allows demand response aggregators (DRAs) to procure DR from consumers and sell it to purchasers. The aggregator obtains DR from the proposed price and incentive-based DR programs. On the other side, the DR outcome is sold to purchasers through the proposed agreements, namely fixed DR contracts and DR options.

Different algorithms have been proposed by researchers for scheduling resources [24, 25, 26] under differing constraints. For instance, scheduling wind resources and storage devices [27, 28, 29], combined with scheduling of generations and DR resources [30, 31]. A robust optimization scheduling framework was proposed [32] to derive an optimal unit commitment decision in systems with high penetration of wind power incorporating demand response programs as well as bulk energy storages in cooptimized energy and reserve markets. Samimi et al. [33] proposed a new economical/environmental operational scheduling method based on sequential day-ahead active and reactive power markets at distribution level to dispatch active and reactive powers in distribution systems with high penetration of distributed energy resources. Faria [34] focused on demand response programs and distributed generation resources management by a virtual power player that aims to minimize operation costs under consumption shifting constraints. Wang et al. [35] formulated a cost minimization problem to intelligently schedule energy generations for microgrids equipped with unstable renewable sources and combined heat and power (CHP) generators. To cope with the uncertain nature of net demand and heat demand, a new flexible uncertainty model was developed which allows the net demand and heat demand distributions to fluctuate around their reference distributions. In [36] a game theoretical demand response program was proposed to determine the optimal hourly incentive to be offered to customers who sign up for load curtailment. In that work, the fuel costs of the generators in the power system and emissions cost are minimized.

Aghajani et al. [37] proposed a multi-objective energy management system in order to optimize micro-grid performance in the short-term in the presence of wind and solar renewable energy sources with a randomized simulated natural behavior. In this research, to implement DR scheduling, incentive-based payment are offered as packages of price and DR quantity collected by Demand Response Providers (DRPs). A methodology was presented in [38] to evaluate and quantify the economic parameters (costs and benefits) attached to customer electricity consumption by analyzing the service provided by the different "pieces" of absorbed electricity. This method helps the customers to integrate in flexible distribution systems and offers the necessary economical parameters for them to integrate in electricity markets. A novel air conditioning system was presented in [39] with proactive demand control for daily load shifting and real time power balance in the developing smart grid. Its purpose is to effectively utilize the demand response potentials of buildings. An optimal integrated participation model of wind and PV energy including demand response, storage devices, and dispatchable distributed generations in microgrids or virtual microgrids was propsed in [40] to increase their revenues in the intra-market. This market is considered 3-7 h before the delivered time, so that the amount of the contracted energy could be updated to reduce the produced power deviation of microgrid. Morais et al. [41] proposed the participation of plug-in hybrid electric vehicles in fuel shifting demand response programs. The proposed services are fuel shifting and fuel discharging. These programs are included in an energy resources management algorithm which integrates the management of other resources.

A control strategy was developed in [42] to provide immediate and stepped power demand reduction through shutting chiller(s) down when requested. The primary control objective of the developed control strategy is to restrain the building indoor temperature rise as to maintain indoor thermal comfort within certain level during the DR event. This research presented an investigations on the power demand alternation potential for buildings involving both active and passive cold storages to support the demand response of buildings connected to smart grids. A stochastic energy procurement problem (SEPP) was proposed by Nojavan and Aalami [43] for large electricity consumer with multiple energy procurement sources (EPS) considering the effects of demand response program and energy storage system. The EPSs contain power market, bilateral contracts, micro-turbines, and 
renewable energy sources. Meanwhile, the uncertainty models of market price, load and renewable output power are considered in the SEPP formulation. Demand response and robust stochastic theory were introduced in [44] to build a stochastic scheduling optimization model to relieve the influence of wind power uncertainty on power system operation. Price-based demand response and incentive-based demand response were introduced to build demand response model. In [44] the demand response revenue functions was constructed for electric vehicle customers, business customers, industry customers and residential customers. Furthermore, robust stochastic optimization theory was introduced to build a wind power consumption stochastic optimization model. A methodology was presented in [45] for the identification, analysis and comparative assessment of the handicaps which nowadays prevent the higher implementation of DR in the electricity market. Genc [46] investigated wholesale demand response to hourly price movements in the Ontario wholesale electricity market using detailed generator and market level data. Genc calculated hourly market power measures such as the Lerner Index and the Residual Supplier Index, which are utilized in a Cournot competition model to structurally estimate price elasticity of demand during peak hours of days, seasons and years.

Much work has been completed in the field of finding the equilibrium in power electricity markets. Several types of market equilibria approaches, such as Cournot [47], Conjectural Variation Equilibria (CVE), Supply Function Equilibria (SFE) or Conjectured Supply Function Equilibria (CSFE) have been used to model electricity markets for the medium and long term. The existence and uniqueness of CSFE was analyzed in [48] for both elastic and inelastic demands. Dolmatova et al. [49] examined existence of a SFE in the model and convergence of the best response dynamics to this equilibrium. They proved the dynamics converges to the SFE for a duopoly, but in general the SFE and the strong best response do not exist in the model. Diaz et al. [50] proposed an algorithm to compute CSFE. In this algorithm, the competitors' strategies for each generator are initially unknown (both slope and intercept) and endogenously computed by this iterative algorithm. Soleymani [51] described a method for analyzing the competition among transmission-constrained Generation Companies (GENCOs) with incomplete information. The proposed methodology employed the SFE for modeling a GENCO's bidding strategy in an energy market and uses an expected payment function to construct a bidding framework in the reactive power market. Holmberg [52] showed valid SFE can be calculated by means of a shooting algorithm that combines numerical integration with an optimization procedure that searches for an end-condition. Holmberg and Newbery [53] showed how welfare losses depend on the number of firms (of a gametheoretic model) in the market and their asymmetry. A new enhanced bat-inspired algorithm was presented in [54] to find out linear SFE of GENCOs in a network-constrained electricity market where they have incomplete information about other rivals. In that work, the social welfare maximization is applied to clear the market and a nodal pricing mechanism is utilized to calculate the GENCO's profit. Rezaei and Kalantar [55] devised and employed a novel methodology on the basis of Nash equilibria to select the best compromise solution from the generated Pareto front. They formulated a frequency security constrained energy management system for an islanded microgrid. A cost effective solution is proposed in [56] to make use of communication technologies to improve DR system integration and interoperability. The system utilizes open standards and optimization models for resource planning using the given dynamic pricing. An approach was presented in [57] to predicted demand response availability for end-use loads for 2020. Moreover, an annual hourly load profile for each of balancing authority areas is provided. A model of uncertain flexibility and associated causes are presented in [58]. Two case studies are given in the paper to discuss the causes and magnitude of uncertainty.

We have developed a model to optimize individual storage device control in response to price signals as a function of consumer defined cost or comfort parameters. In our model, we combine individual device nodes into aggregate microgrid groups simplifying agent interaction by adding a layer of abstraction. The autonomy of each aggregate group to optimize its own pooled resources is desirable, especially with the presence of distributed, intermittent, renewable energy generation. Each group first makes most efficient use of local resources, then participates in the larger grid market based on the supply or demand of its pooled resources. Each smart agent involved is in charge of controlling the resources of its own partition aggregate. We determined that there are two factors each smart agent must maintain for optimum performance. They must simultaneously maximize cost savings for all participants while maintaining the minimum comfort parameters set by consumers. Really, the only control these smart agents have is to switch on and off controllable loads within their pool-thermal, mechanical, or chemical storage - so, of course, the output of this mathematical model is in terms of change in controllable load. Each smart agent optimizes load switching within its own pool.

In order to quantify optimum parameters based on cost, market analysis was carefully considered. There are several imperfect competition models available to quantify market conditions including: the Cournot, supply function equilibrium (SFE), Bertrand, and monopoly models [59]. The monopoly model was unsuitable for conditions with consumer owned distributed renewable energy generation throughout the grid. The Bertrand model assumes a market that has producers that have identical marginal cost curves. This is ideal for modeling several competing large utilities however, not representative of distributed renewable generation that has widely different marginal cost of production. This leaves us with the Cournot and supply function equilibrium models which have flexibility to account for different marginal costs of production between competing firms on the market. There exists contention as to which model is more effective. The Cournot models costs based on demand while the SFE uses price. Simulations using both models have been performed and the results of the comparative analysis are shown in the following sections. With effective means of communication and control, and the ability to process large amounts of data, mathematical models provide a means to quantify and implement grid optimization. We will describe the combination of these 
methods into an effective strategy for DR. The overall structure of demand response market is shown in figure 1. The GENCO or utility performs an optimization for power generation using optimal power flow to find the optimal cost for each amount of output power. The Locational Marginal Price (LMP) is announced to the participating demand response aggregators. Based on the given price signal, the aggregators operate their resources to maximize their revenue. These resources include water heater, $\mathrm{AC}$ and residential battery storage. The gadgets for controlling these devices are described in the appendix B.

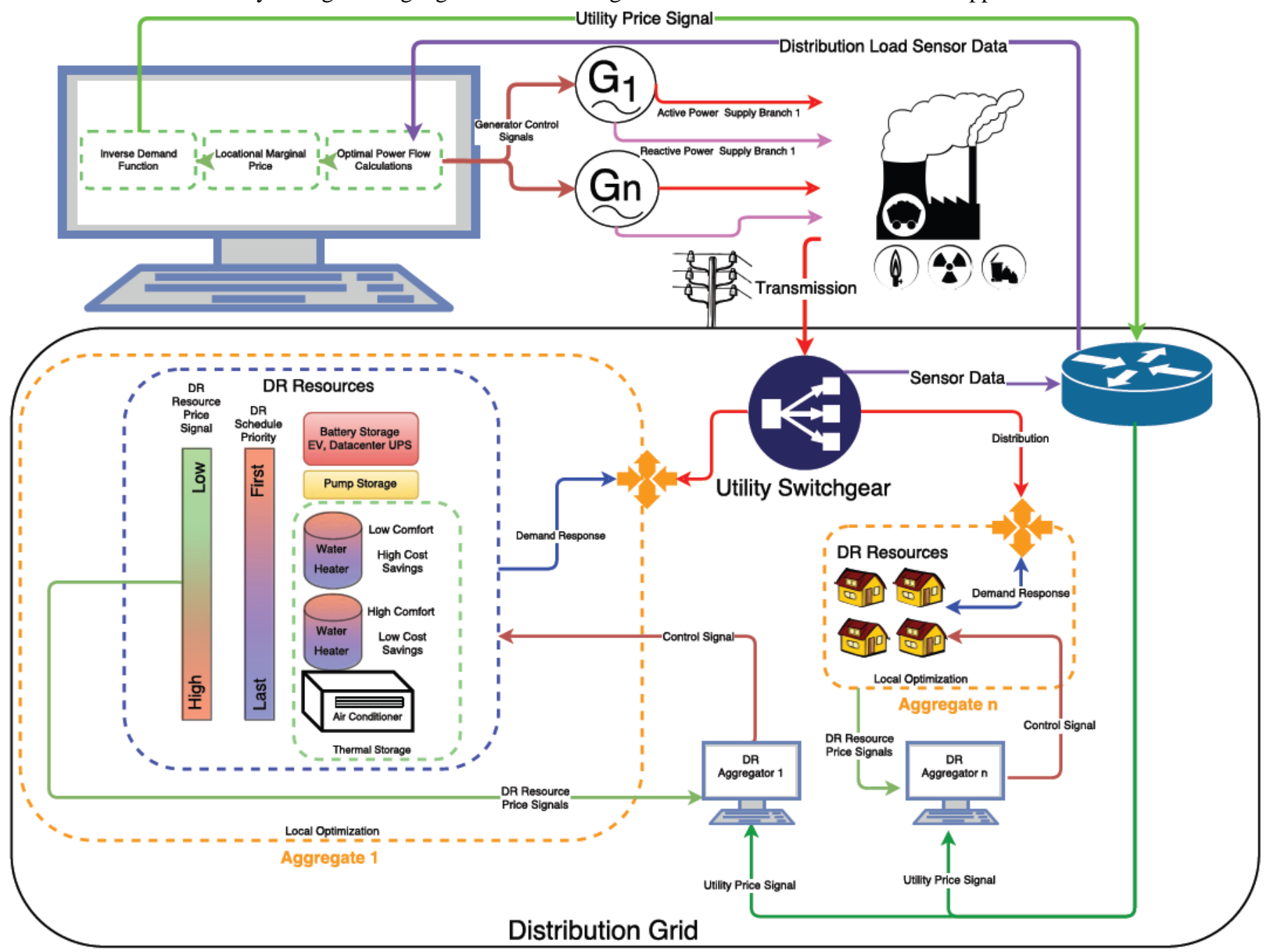

Figure 1: Aggregate control and local optimization of DR resources in the interactive grid market

\section{DR MARKET}

\section{A. Optimal Power Flow}

The standard AC Optimal Power Flow (OPF) equations minimize cost of overall real and reactive power injections by a generator [60]. These optimal values of active and reactive power for each generator are calculated using the methods described by Zimmerman. The cost functions used to arrive at the optimal active and reactive power generation for each individual generator are constrained by:

- Balance of power generated with power consumed - active and reactive.

- The limit of power flow at each end — to and from — of each supply branch (branch flow limits are calculated using nonlinear functions of bus voltage angles and magnitudes).

- The set value for maximum deviation from reference voltage angle.

- The minimum and maximum allowable voltage at each bus.

- Maximum and minimum amount of power generation by each generator.

The minimization problem can be expressed as follows: 
$\underset{\Theta, V_{m}, P_{g}, Q_{g}}{\operatorname{Min}} \sum_{i=1}^{n_{g}} f_{P_{i}}\left(p_{g_{i}}\right)+f_{Q_{i}}\left(q_{g_{i}}\right)$

$f_{P_{i}}\left(p_{g_{i}}\right)$ and $f_{Q_{i}}\left(q_{g_{i}}\right)$ are cost functions of active and reactive power generation for generator $i$, respectively. $P_{g}$ and $Q_{g}$ are sets of possible values for active and reactive generations at each respective generator. $\Theta$ is the set of bus voltage angles and $V_{m}$ shows the set of voltage magnitudes at each bus. $n_{g}$ is the number of generators.

From a DR perspective, we can view the total energy of the system as remaining constant. The sum of the power contribution from demand response and contribution from power generators remains constant. The amount of contribution from one or the other, for any given time period, may be adjusted provided that the other is adjusted complementarily so that their sum remains constant. We define the location marginal price (LMP) as the cost of power for each pair of values of DR and generator contributions. The LMP is proportional to the power draw and therefore inversely proportional to the amount of DR contribution. For any given time period with its own unique set of values for power draw and available DR resources, OPF is performed at each possible level of DR contribution to obtain an LMP value at each point. Performing, the method of least squares on the output to obtain linear regression, we arrive at an approximation for inverse demand function with an example set of values for IEEE 30 bus test case.

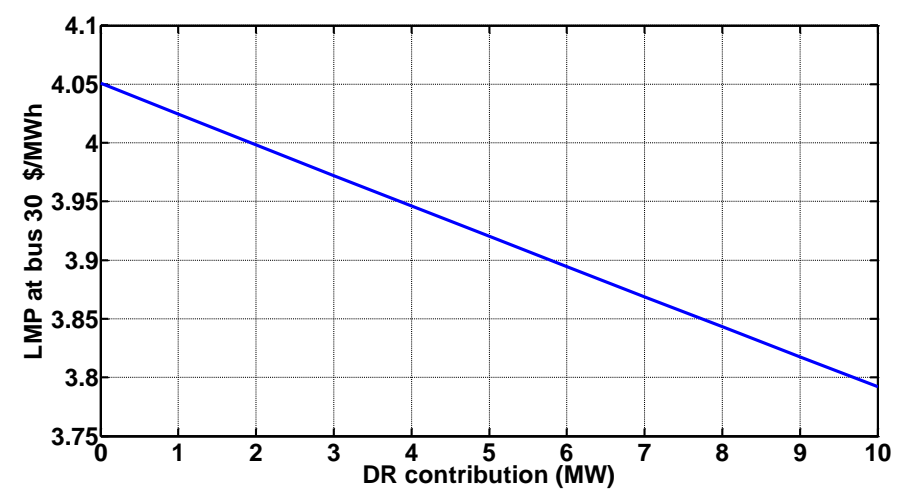

Figure 2: Example of LMP curve and inverse demand function on IEEE 30 bus test case.

The calculated inverse demand function may be expressed as:

$\pi(\Delta L)=\Phi-\xi \Delta L$

Where $\pi$ is the price given per unit of demand response received, $\Phi$ is the offset of inverse demand function, $\Delta L$ is the demand response contribution, and $\xi$ shows the rate of change of price with respect to demand response contribution.

\section{B. Demand response market}

Financial incentive is offered to motivate consumers to actively participate in the management of load distribution through demand response. As mentioned previously, increases in the presence of intermittent renewable energy generation throughout the grid make the ability to effectively distribute loads throughout the day even more important. The DR market consists of two entities: a utility who acts as a buyer of demand response, and distribution system consumers that own demand response capable equipment who act as sellers. The product being purchased and sold is demand response load. A nice feature of this model is that the exact amount of demand for the product is known, which is given by equation 1. Each consumer therefore bids a price to sell their share of the available demand for distribution grid load adjustment in the form of demand response. The final market price depends on the bids of all participants. A layer of abstraction can be added by breaking the grid up into microgrids, each controlled by a DRA. The DRA optimizes its own section of the grid and bids on the demand response market on behalf of its available units. The DRA optimizes its available resources and estimates the market price to place a bid that will maximize its profit in the demand response market. Several assumptions are made in developing this model:

- No party has the power to influence the market.

- Residential DRAs have nearly equal capacity of DR.

- No single DRA controls a significantly large proportion of the overall DR quantity.

At each time step, each house bids a discomfort price with the goal of maximizing its profit without cooperating with other agents. The utility buys the aggregate amount of load adjustment DR from DRAs with bids within the determined acceptable range in order to minimize its purchasing costs. Thus an equilibrium point is found and the market share is cleared. The utility is able to minimize cost of turning on peak generators by purchasing demand response for correction from non-optimal load values. 
Such interaction between entities is modeled by the game theoretical concept as a non-cooperative game. The Nash (market) Equilibrium is obtained for imperfectly competitive environments using Cournot and supply function equilibrium models. In the DR market, players are competing with each other to sell the proposed amount of DR resources. Each player provides a cost function to the market operator considering the constraints. The market is then cleared using two given approaches, Cournot and SFE. The inverse demand function is obtained by performing an optimal power flow in which the energy price for different amount of DR contribution is obtained.

The uniqueness of the research work is creating a market in which DRAs can propose their cost function considering the constraints. After the market is cleared, each DRA needs to provide the DR amount specified in the market. The method of deriving cost function is also presented in the appendix A. It is shown that a quadratic cost function can describe the behavior of DR aggregator. The DR aggregator can optimize its assets to optimally procure the amount of DR value cleared in the market.

\section{Cournot model for two competing DRAs}

In the Cournot model, the DRAs are competing with each other independently to sell their share of DR. In determining the cost attached to providing demand response, the DRA uses the quadratic cost function as shown below. Appendix A proves how a discomfort function for DR of a house can be modeled with a quadratic function. The discomfort cost associated with deploying each individual DR resource unit's available DR load is determined by the unit's system state - temperature in the case of a water heater - and the unit's comfort setting. Using the inverse demand function as a reference point, the maximum price per unit of demand response is $\pi$ (equation 2). Assuming that this is the highest price that available demand response load may be sold for, units set at the maximum comfort setting within the aggregate will take on this value for discomfort cost. Since each individual house's discomfort functions is quadratic, the sum of the DR quantity of the houses will also produce a quadratic cost function for the aggregator:

$$
C_{i}\left(\Delta L_{i}\right)=\frac{1}{2} u_{i} \Delta L_{i}^{2}+v_{i} \Delta L_{i}
$$

Where $C_{i}$ is cost of providing demand response for aggregator $i(\$ / \mathrm{kW})$. The aggregator's cost function includes the sum of discomfort costs of individual houses as well as the profit for the aggregator. In order to find the coefficients of the cost function, the aggregator needs to perform an optimization among its customers to come up with an optimal cost curve. Each customer provides a quadratic discomfort cost function with known coefficients according to Appendix A to the DRA within its available power (equation 4). The overall cost per unit of DR for every DRA becomes the output of the minimization of the sum of the discomfort cost of each customer's demand response. Solving equation 5 for a given set of DR values produces the optimal cost curve for the aggregator:

$$
\begin{aligned}
& C^{d c}\left(\Delta L_{h_{i}}\right)=\frac{1}{2} u_{h_{i}} \Delta L_{h_{i}}{ }^{2}+v_{h_{i}} \Delta L_{h_{i}} \\
& C_{i}\left(\Delta L_{i}\right)=\min \left\{\sum_{i \in A_{b}} C^{d c}\left(\Delta L_{h_{i}}\right)\right\}
\end{aligned}
$$

Subject to the following constraints:

$$
\begin{aligned}
& \Delta L_{h_{i, \min }} \leq \Delta L_{h_{i}} \leq \Delta L_{h_{i, \text { max }}} \\
& \sum_{i \in K} \Delta L_{h_{i}} \leq S_{T_{K}}^{\max }
\end{aligned}
$$

$C^{d c}\left(\Delta L_{h_{i}}\right)$ is the amount the aggregator pays house $i$ for its DR contribution $\left(\Delta L_{h_{i}}\right)$ and $S_{T_{K}}^{\max }$ is the nominal power of transformer at lateral distribution branch $k$. [ $\left.\Delta L_{h_{i, \min }}, \Delta L_{h_{i, \max }}\right]$ is the range of possible DR contributions of house $i$. In addition to the above constraints a voltage regulation constraint can also be embedded in the above formulation to ensure that power flow resulting from DR providers will not violate voltage regulation constraints along the distribution system. This is especially important in the presence of large amounts of intermittent renewable energy generation which show stochastic trends. Consider the simple radial system [61]: 


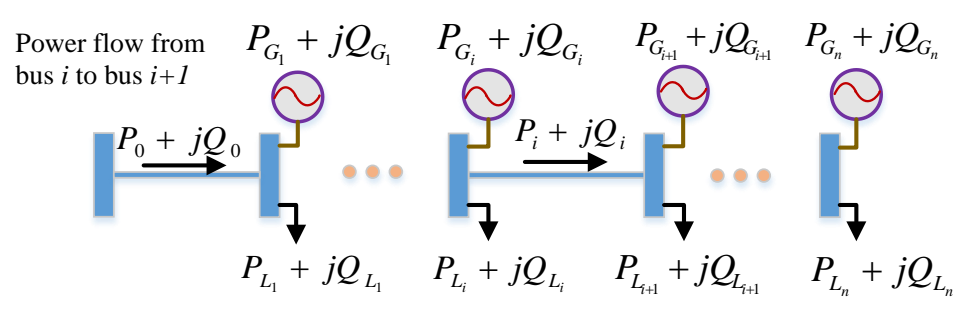

Figure 3: Schematic diagram of example radial distribution system.

$P_{G_{i}}, Q_{G_{i}}$ are active and reactive power generated by renewable energy sources and $P_{L_{i}}, Q_{L_{i}}$ are active and reactive loads at house $i$, respectively. The power flow and voltage for the above radial distribution system (ignoring line losses) are given by equations (6), and (7) :

$P_{m}=\sum_{k=m+1}^{N_{b}} P_{L_{k}}-\sum_{k=m+1}^{N_{b}} P_{G_{k}}-\sum_{k=m+1}^{N_{b}} \Delta L_{k}$

$V_{m}^{2}=V_{m-1}^{2}-2\left(r_{m} P_{m-1}+x_{m} Q_{m-1}\right)+\frac{\left(r_{m}^{2}+x_{m}^{2}\right)\left(P_{m-1}^{2}+Q_{m-1}^{2}\right)}{V_{m-1}^{2}}$

$m=1, \ldots, N_{b}$

$P_{m}$ is power available at bus location $m . r_{i}, x_{i}, V_{i}$ are resistance, reactance, and voltage parameters at bus $i$ and $N_{b}$ is the number of busses in the radial distribution grid.

Starting with $N=1$ and assuming that the service transformer voltage $V_{0}$ is given, $V_{1}$ to $V_{N}$ are obtained by iteration of equation (7). The nonlinear constraint of the optimization problem which addresses the voltage control constraint is given by:

$\sum_{i}\left(V_{i}-1.0\right)^{2}=0$

In the Cournot model, each DRA analyzes the availability of its DR resources at each time step, and bids a quantity of load reduction, $\Delta L_{i}$. The profit of aggregator $i(\$ / \mathrm{h}), \Omega_{i}$, is the difference between what the utility is willing to offer and the discomfort cost of the DRA:

$\Omega_{i}=\pi(\Delta L) \Delta L_{i}-C_{i}\left(\Delta L_{i}\right)$

Setting the partial derivative of each DRA, with respect to its contribution in demand response load, equal to 0 gives the optimal value:

$\frac{\partial \Omega_{i}}{\partial \Delta L_{i}}=\pi(\Delta L)-u_{i} \Delta L_{i}-v_{i}+\Delta L_{1} \frac{d \pi}{d \Delta L} \frac{d \Delta L}{d \Delta L_{i}}=0$

$i=0,1, \ldots, m$

Expanding the above equations for $m$ DRAs yields a set of equations:

$\Phi-\xi \sum_{i \in A_{b}} \Delta L_{i}-\left(u_{i}+\xi\right) \Delta L_{i}-v_{i}=0 \quad i=1, \ldots, m$

The value of DR contribution in load for each DRA is calculated from the above set of equations. Each DRA then optimizes its resources based on the amount of DR it has won on the market.

By finding the optimal costs at for each for each possible value of DR up to the maximum available from registered customers in the circuit gives us a list of points for our optimal cost curve. The method of least squares is applied to fit a quadratic cost function is fit to the points. This determines the cost function's coefficients (equation 5).

\section{Supply function equilibrium}

It has been proposed that the Cournot model may have several limitations. The amount of power produced by each aggregator in the Cournot model is very sensitive to the bidding price $\left(\varepsilon_{i}\right)$ and small changes in the cost coefficient lead to sharp changes in the amount of demand repose quantities [8]. Some claim that market behavior would be better predicted by a SFE model, however this is more computationally intensive because both supply and cost functions must be determined. In contrast to the 
Cournot model the SFE considers that the amount of DR provided by each aggregator is dependent upon the market price and total DR quantities. Using these values as input, we may arrive at the Nash equilibrium point shown by:

$\Delta L(\pi)=\sum_{a} \Delta L_{a}$

The profit for each aggregator is the difference between the revenue from DR sold and the discomfort cost of implementation:

$\Omega_{i}=\pi \Delta L_{i}-C_{i}\left(\Delta L_{i}\right)$

$\Omega_{i}=\pi\left(\Delta L(\pi)-\sum_{-i} \Delta L_{-i}(\pi)\right)-C_{i}\left(\Delta L(\pi)-\sum_{-i} \Delta L_{-i}(\pi)\right)$

Taking the first derivative of each aggregator's profit with respect to the price of DR, the aggregator's contribution to the total DR is calculated as follows:

$\Delta L_{i}(\pi)=\left(\pi-\frac{d C_{i}\left(\Delta L_{i}\right)}{d \Delta L_{i}}\right)\left(-\frac{d \Delta L}{d \pi}+\sum_{-i} \frac{d \Delta L_{-i}(\pi)}{d \pi}\right)$

We express the supply and cost function as affine quadratic functions [62]:

Supply:

$\Delta L_{i}(\pi)=\beta_{i}\left(\pi-\alpha_{i}\right) \quad \forall i \in A_{b}$

Cost:

$C_{i}\left(\Delta L_{i}\right)=\frac{1}{2} u_{i} \Delta L_{i}^{2}+v_{i} \Delta L_{i} \quad \forall i \in A_{b}$

Coefficients $\mathrm{u}$ and $\mathrm{v}$, for the cost function are determined using the same methods previously described for the Cournot model. Once $\mathrm{u}$ and $\mathrm{v}$ are known, we may solve for alpha and beta in the supply function. Substituting the demand (equation 4), supply (equation 18) and cost (equation 19) functions into equation 17, then using the methods described by [63] we arrive at Green's equation:

$\beta_{i}=\left(1-u_{i} \beta_{i}\right)\left(1 / \xi+\sum_{j \in A_{b}, j \neq i} \beta_{j}\right) \quad \forall i \in A_{b}$

$\alpha_{i}=v_{i}$

The solution is calculated beginning with $u_{a}=1 / \beta_{a}$ in the first iteration. Each aggregator updates its value of $\beta_{a}$ given the values of $\beta_{i}$ for the other firms from the previous iteration. The sequence will converge to one non-negative solution.

\section{NUMERICAL EXAMPLE AND COMPARISON SFE vs COURNOT}

\section{A. Cournot model Results (Two DRAs)}

OPF is performed on bus number 30, IEEE 30 bus test case. The LMP for electrical energy at different levels of DR contribution is given shown in Fig. 5:

The equation for inverse demand function obtained by OPF becomes:

$\pi=4.05-0.026 \Delta L \quad \$ / M W h$

Contribution of each DRA is obtained by equation 13:

$\Delta L_{1}=0.5338 M W$

$\Delta L_{2}=.5883 M W$

The market price of about $4 \$ \mathrm{MWh}$ is calculated by summing up the DR contributions and plugging into the inverse demand function.

B. SFE results (Two DRAs)

Suppose that DRA1 and DR2 have the following cost functions:

$C_{1}\left(\Delta L_{1}\right)=3.186 \Delta L_{1}^{2}+0.63 \Delta L_{1}$

$C_{2}\left(\Delta L_{2}\right)=3 \Delta L_{2}^{2}+0.5 \Delta L_{2}$ 
From equation 18, we obtain the following values for each aggregator's DR contribution:

$\Delta L_{1}=0.5338 M W$

$\Delta L_{2}=.5883 M W$

The market price is $\sim 4 / M W h$.

\section{Comparison}

The contribution of each aggregator using Cournot and SFE market models are calculated and compared with each other in figure 6. The market price shown in Fig. 5 shows convergence of both methods as the elasticity of the price goes higher. For low price elasticity values, the Cournot model gives a lower price compared with SFE model. In the inverse demand function in Fig. 5 where the price elasticity is high, both models produce the same result. The first and second aggregator DR contributions also reach the same value at the obtained price elasticity value of 38.94 which is the inverse of the slope of the line in Fig. 5.
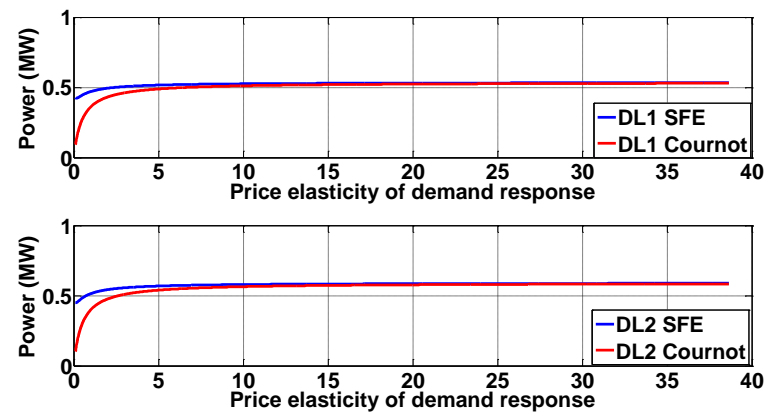

Figure 4: DRA 1 and 2 contributions using Cournot and SFE models

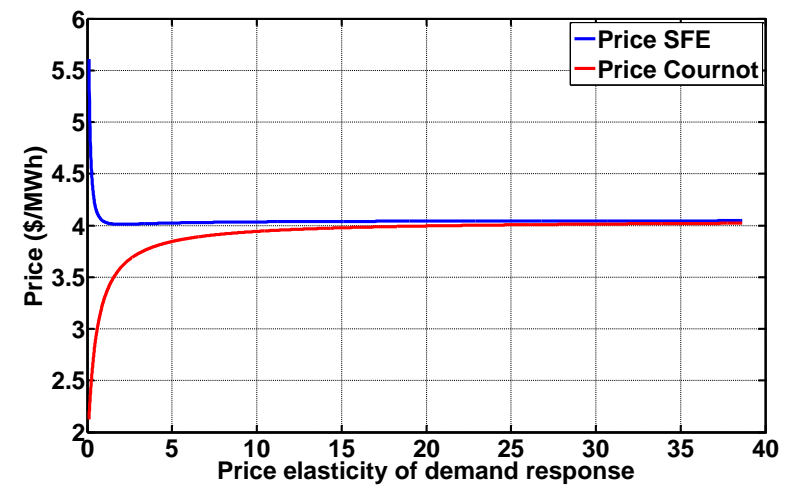

Figure 5: Market price using two models

\section{DR optimization results}

Suppose aggregator 1 wants to optimize 0.5338 MW of DR from the available resources in the circuit. It can optimize it based on the cost function provided by each house and also the constraints given in relations 7 and 10. Using coefficients and maximum DR capacity from table 1, the aggregator determines the contribution of each house or provider (DRP) shown in Fig. 6.

Table 1: Data for DRPs of aggregator 1

\begin{tabular}{cccl}
\hline DRP number & $u_{h_{i}}$ & \multicolumn{1}{c}{$v_{h_{i}}$} & \multicolumn{1}{c}{$u b_{h_{i}}$} \\
\hline $\mathbf{1}$ & 58.1 & 0.63 & 0.0588 \\
\hline $\mathbf{2}$ & 24.4 & 0.63 & 0.14 \\
\hline $\mathbf{3}$ & 35.88 & 0.63 & 0.0952 \\
\hline $\mathbf{4}$ & 24.9 & 0.63 & 0.1372 \\
\hline $\mathbf{5}$ & 35.88 & 0.63 & 0.0952 \\
\hline $\mathbf{6}$ & 45.2 & 0.63 & 0.0756 \\
\hline
\end{tabular}




\begin{tabular}{cccl}
\hline $\mathbf{7}$ & 30.5 & 0.63 & 0.1120 \\
\hline $\mathbf{8}$ & 27.11 & 0.63 & 0.1260 \\
\hline $\mathbf{9}$ & 34.86 & 0.63 & 0.098 \\
\hline $\mathbf{1 0}$ & 25.42 & 0.63 & 0.1344 \\
\hline
\end{tabular}

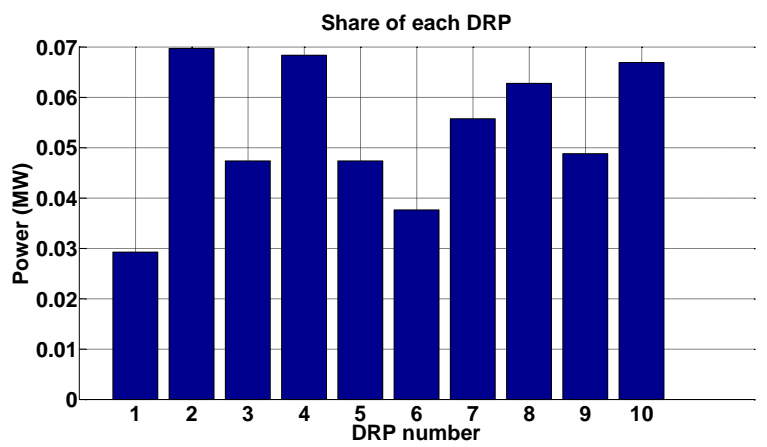

Figure 6: Contribution of each DRP obtained by optimization of resources by aggregator 1

\section{E. Sensitivity analysis for cost function coefficients}

In order to better understand the impact of the discomfort cost function coefficients of houses on the amount of demand response provided and also the revenue of each house, we perform a sensitivity analysis on the cost coefficients of a sample house. The first coefficient of house 1 is increased from 1 to 58 in one step increments.

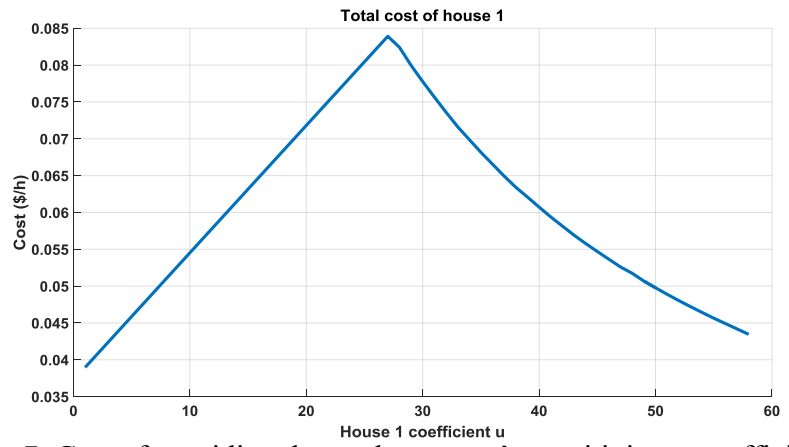

Figure 7: Cost of providing demand response's sensitivity to coefficient $u$

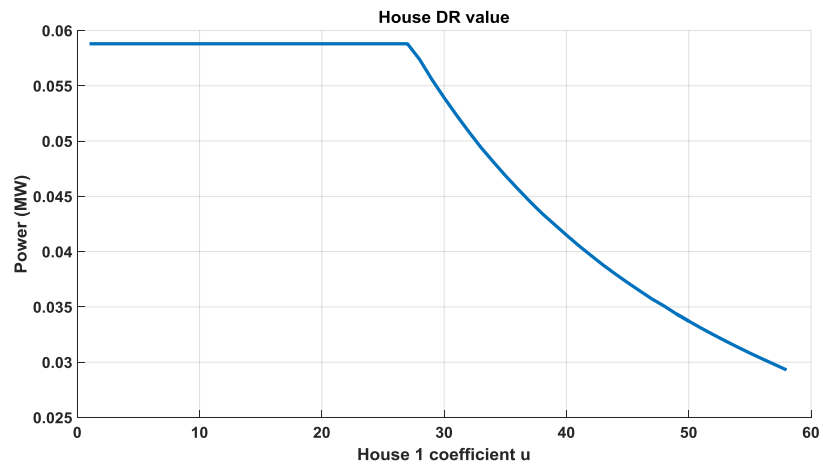

Figure 8: Provided demand response's sensitivity to coefficient $u$

As shown in figures 7 and 8, the amount of DR is constant up to a certain value of $u$ coefficient. The corresponding cost of providing DR is increasing accordingly. Therefore, for the same amount of DR, it is preferable to operate the devices at minimum cost. If the houses may bid for the DR provided, a market similar to DR market can be realized for calculating each house's share. The players in this market may have complete or incomplete information about their competitors and thus their bidding strategy will change accordingly. 


\section{CONCLUSION}

In this paper, demand response resources are managed using two different approaches. In the first approach, a direct load control method is used. The goal of DLC is to flatten or follow a reference load curve by optimal switching of water heaters or ACs. In the second approach, a market mechanism is proposed in which demand response aggregators can sell the available amount of DR to the utility. The utility determines an inverse demand function at each node which describes the cost of providing power at that node. This cost function is calculated by a continuous optimal power flow with differing DR contributions. Each aggregator at a node calculated the optimal cost function based on the available resources. The market is cleared using two models, Cournot and SFE. The price as well as contribution of each aggregator is compared for different price elasticities. It is shown that at high price elasticity values, both models produce the same result.

\section{ACKNOWLEDGEMENT}

This dissertation is sponsored by US National Science Foundation under award number: 1310709.

\section{APPENDIX A}

A high percentage of demand response employs water heaters and residential energy storage devices. Thus, the discomfort function of demand response is approximated by the summation of the discomfort functions of these two devices with appropriate coefficients.

\section{A. Battery Model}

For the battery, the function we chose is a variant of the widely used logarithmic barrier function, used as a penalty function in interior point methods [64]:

$$
C_{h_{i}}^{\text {bat }}\left(\Delta E_{h_{i}}\right)=-a_{h_{i}} \log \left(1-\frac{\Delta E_{h_{i}}}{B}\right)
$$

Where $C_{h_{i}}^{b a t}$ is cost of the battery in house $i$ as a function of stored energy, $a_{h_{i}}$ is a pricing coefficient determined by the utility to give higher prices during peak-hours, $\Delta E_{h_{i}}$ is the total load (kWh), $B$ is a parameter that we introduce to give cost values very close to the values given by a quadratic cost function- it also serves as the maximum typical value for $\left|\Delta E_{h_{i}}\right|$. The relation between our proposed cost function and the quadratic can be understood from its Taylor expansion. Since $\Delta E_{h_{i}} / B<1$, the Taylor series expansion is:

$$
\begin{aligned}
& C_{i}^{b a t}\left(\Delta E_{h_{i}}\right)=-a_{h_{i}} \log \left(1-\frac{\Delta E_{h_{i}}}{B}\right) \\
& \approx-a_{h_{i}}\left(-\frac{\Delta E_{h_{i}}}{B}-\frac{\Delta E_{h_{i}}^{2}}{B^{2}}\right)=\frac{a_{h_{i}} \Delta E_{i}}{B}+\frac{a_{h_{i}} \Delta E_{i}^{2}}{B^{2}}
\end{aligned}
$$

For our purposes, we assume that the load data is available from measurement devices for every 15 minute period. It is also assumed that the load is constant for each 15 minute period. The quadratic cost function of the battery can be rewritten as a function of power $\left(\Delta L_{h_{i}}\right)$ :

$$
C_{h_{i}}^{b a t}\left(\Delta L_{h_{i}}\right)=\frac{a_{h_{i}}\left(0.25 \Delta L_{h_{i}}\right)}{B}+\frac{a_{h_{i}}\left(0.25 \Delta L_{h_{i}}\right)^{2}}{B^{2}}
$$

In order to obtain the coefficients $a_{h_{i}}$ and $B$, two factors should be considered: the electricity price of the grid (for charging the battery) and the capital and maintenance costs of the battery. Thus, the cost of the battery of house $i$ for charging the power as a function of $\Delta L_{h_{i}}$ is:

$C_{h_{i}}^{\text {bat }}\left(\Delta L_{h_{i}}\right)=C_{h_{i}}^{\text {grid }}\left(\Delta L_{h_{i}}\right)+C_{h_{i}}^{C \& M}\left(\Delta L_{h_{i}}\right)$

Where $C_{h_{i}}^{\text {grid }}\left(\Delta L_{h_{i}}\right)$ is the cost which house $i$ pays to the utility to sell the electricity and charge its own battery with an amount of power of $\Delta L_{h_{i}}$. This cost comes from procuring power from the grid which is linked to the demand. Also, $C_{h_{i}}^{C \& M}\left(\Delta L_{h_{i}}\right)$ is the contribution of capital and maintenance costs of the battery of house $i$ for power of $\Delta L_{h_{i}}$. The coefficients $a_{h_{i}}$ and $B$ can be 
obtained using equation (22). The discomfort function of the battery in house $i$ as a function of $\Delta L_{h_{i}}, C_{h_{i}}^{d c, \text { bat }}\left(\Delta L_{h_{i}}\right)$, is the money which house $i$ is willing to accept as payment for the lessened comfort associated with participation in the demand response program. This discomfort function is based on the contract between the aggregators and participants which must be greater than or equal to the cost function of the battery:

$C_{h_{i}}^{d c, \text { bat }}\left(\Delta L_{h_{i}}\right) \geq C_{h_{i}}^{\text {bat }}\left(\Delta L_{h_{i}}\right)$

\section{B. Water heater}

For water heaters, the discomfort function of house $i, C_{h_{i}}^{d c, w h}$, is modeled by a linear function of deviation from the minimum of the temperature comfort range. If $\left[T_{\min }, T_{\max }\right]$ is the temperature range of water considered comfortable, the discomfort function of the water heater is:

$$
C_{h_{i}}^{d c, w h}\left(\Delta L_{h_{i}}\right) \propto \frac{\Delta T}{\Delta t}
$$

Where $\Delta T=T_{\min }-T_{d c}$. The parameter $T_{d c}$ is the water temperature in discomfort zone. For example, in the contract between house $i$ and aggregator, if participant claims $2 \$ /^{\circ} \mathrm{C} \cdot h$, it means the participant gets $2 \$$ if the water is $1^{\circ} \mathrm{C}$ colder than $T_{\text {min }}$ for one hour. The discomfort cost function for water heater depends on the temperate change which will be regulated by drawing power from the grid. Thus, the water heater acts as a smart load to minimize the cost while satisfying the temperature constraints. A thermodynamic model of water heaters is presented in [65] as following equation (25):

$$
M C \frac{\Delta T}{\Delta t}=P_{e}+P_{c w}-P_{h w}-P_{l o s s}
$$

Where $M$ is the mass of water in the tank, $C$ is the specific heat, $P_{e}$ is the thermal power supplied by the heating element (electrical power), $P_{c w}$ and $P_{h w}$ are water inflow and outflow of the tank, and $P_{l o s s}$ denotes the heat transferred to the ambient surroundings. Clearly, the rate of changing the temperature is a linear function of electrical power:

$\left(\frac{\Delta T}{\Delta t} \propto \frac{1}{M C} P_{e}\right)$. Since in our work, the market is cleared for each time interval of $\Delta t=15$ minues, the discomfort function for the water heater of house $i$ (equation 25) can be rewritten as:

$$
\begin{gathered}
C_{h_{i}}^{d c, w h}\left(\Delta L_{h_{i}}\right) \propto \frac{1}{M C} \Delta L_{h_{i}} \\
\text { or } C_{h_{i}}^{d c, w h}\left(\Delta L_{h_{i}}\right)=\frac{\beta}{M C} \Delta L_{h_{i}}
\end{gathered}
$$

Since about $60 \%$ of the load a typical house includes water heaters (or air conditions with the same thermodynamic modelACs), we can use the above discomfort function (equations 24, 27) as an approximation to the total discomfort function for all electrical devices in the houses. The final discomfort function for the demand response of house $i\left(C_{h_{i}}\right)$ will be the sum of battery discomfort function (22) and the water heater discomfort function (27) in equation 28 (or ACs).

$$
C_{h_{i}}\left(\Delta L_{h_{i}}\right)=C_{h_{i}}^{d c, b a t}\left(\Delta L_{h_{i}}\right)+\frac{10}{6} C_{h_{i}}^{d c, w h}\left(\Delta L_{h_{i}}\right)
$$

The final DR discomfort function for house $i$ will be the quadratic function (29):

$$
C_{h_{i}}\left(\Delta L_{h_{i}}\right)=\frac{1}{2} u_{h_{i}} \Delta L_{h_{i}}^{2}+v_{h_{i}} \Delta L_{h_{i}}
$$

Which is employed in this paper. The coefficients of $u_{i}$ and $v_{i}$ can be obtained from the following equations:

$$
\frac{1}{2} u_{h_{i}}=\frac{a_{h_{i}}(0.25)^{2}}{B^{2}}
$$


$v_{h_{i}}=\frac{a_{h_{i}}(0.25)}{B}+\frac{10}{6} \frac{\beta}{M C}$

Coefficient of $\frac{10}{6}$ is added to make an appropriate weigh for all other electrical devices of house $i$, based on the mentioned reason.

\section{APPENDIX B}

Several distributed sensor and control devices and a scalable software testbed were developed at the Renewable Energy Design Lab (REDLab) at the University of Hawaii at Manoa. These hardware devices were designed to be able to both monitor the power of dynamic loads and exert control over them in response to control signals from a central system. In collaboration with our industry partner, Greenpath Technologies, these devices were installed in Lezeti AC/DC hybrid air conditioning units as a pilot implementation shown in Figure 9. In order to enhance cybersecurity, these devices were designed to communicate over encrypted channels using existing network infrastructure. The devices are designed to communicate wirelessly to create a wireless sensor and actuator network (WSAN). The network is arranged in a cluster topology. Multiple sensor and control devices are connected to a wireless secure smart appliance network (SSAN) gateway positioned within range. The system was designed with the intent of one gateway per residential location to serve all the sensors within the house. The SSAN connects to the residential internet access point. The devices receive forwarded control commands through the SSAN gateway and report back sensor data about power consumption of the dynamic loads. Sensor data is written to the server in processed and agglomerated packages at a $\sim 1 \mathrm{~Hz}$ rate to a relational SQL database.
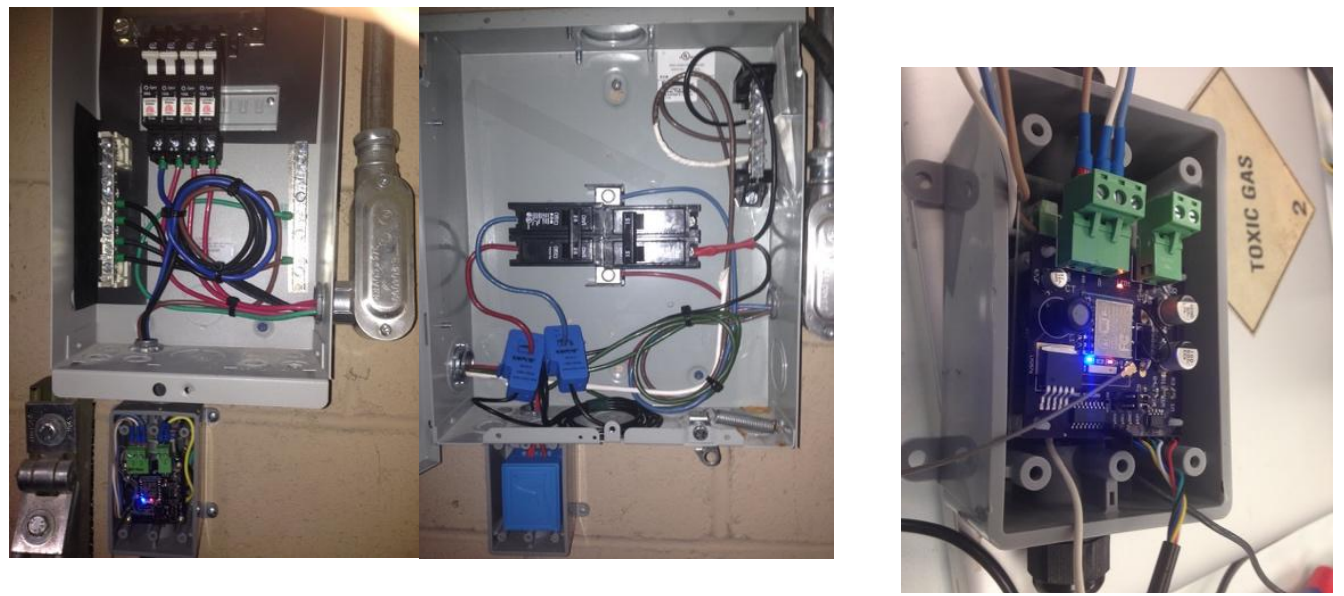

Figure 9: Distributed sensor and control devices installed on site on Lezeti hybrid AC/DC air conditioning system.

In order to both vet the DR distributed sensor and control devices and test the practical application of the proposed method of DR scheduling, a testbed was developed using PSIM power simulation software [66] which was modified to be able to communicate with both other computer terminals acting as DR aggregators and real distributed device nodes over encrypted network channels. The result was an internet hardware in the loop simulation environment (iHILS) explained in detail in [67]. The simulation environment creates a virtual space where grid conditions can be modeled and control algorithms can be implemented as the simulation receives sensor data and sends control signals to distributed hardware. The grid simulation allows detailed monitoring of all points of the systems being modeled and control over the individual elements in the system, which gives flexibility for detailed simulations of grid systems with high penetrations of intermittent renewable energy generation. Simulated nodes give the system scalability without the need for a large scale pilot implementation. Simulated nodes can be monitored within the software itself while real hardware device outputs are automatically recorded through outputs to the network database.

\section{BIBLIOGRAPHY}

[1] C. Chen, J. Wang and S. Kishore, "A Distributed Direct Load Control Approach for Large-Scale Residential Demand Response," IEEE Transactions on Power Systems, vol. 29, no. 5, pp. 2219-2228, 2014.

[2] S. M. Basnet, H. Aburub and W. Jewell, "Effect of demand response on residential energy efficiency with direct load control and dynamic price control," in PowerTech, 2015 IEEE Eindhoven, Eindhoven, 2015. 
[3] F. Zhang, R. D. Dear, C. Candido, "Thermal comfort during temperature cycles induced by direct load control strategies of peak electricity demand management," Building and Environment, vol. 103, pp. 9-20, 2016.

[4] D. Karl Critz, S. Busche, S. Connors, "Power systems balancing with high penetration renewables: The potential of demand response in Hawaii," Energy Conversion and Management, vol. 76, p. 76, 609-619.

[5] P. Siano, D. Sarno, "Assessing the benefits of residential demand response in a real time distribution energy market," Applied Energy, vol. 161, pp. 533-551, 2016.

[6] Q. Wang, C. Zhang, Y. Ding, G. Xydis, J. Wang, J. Østergaard, "Review of real-time electricity markets for integrating Distributed Energy Resources and Demand Response," Applied Energy, vol. 138, pp. 695-706, 2016.

[7] M. Motalleb, M. Thornton, E. Reihani, R. Ghorbani, "A nascent market for contingency reserve services using demand response," Applied Energy, vol. 179, pp. 985-995, 2016.

[8] M. Motalleb, M. Thornton, E. Reihani, R. Ghorbani, "Providing frequency regulation reserve services using demand response," Energy Conversion and Management, vol. 124, pp. 439-452, 2016.

[9] M. Behrangrad, H. Sugihara, T. Funaki, "Effect of optimal spinning reserve requirement on system pollution emission considering reserve supplying demand response in the electricity market," Applied Energy, vol. 88, pp. 2548-2558, 2011.

[10] B. Shen, G. Ghatikar, Z. Lei, J. Li, G. Wikler, P. Martin, "The role of regulatory reforms, market changes, and technology development to make demand response a viable resource in meeting energy challenges," Applied Energy, vol. 130, pp. 814$823,2014$.

[11] M. Rahmani-andebili, "Modeling nonlinear incentive-based and price-based demandresponse programs and implementing on real power markets," Electric Power Systems Research, vol. 132, pp. 115-124, 2016.

[12] P. Zou, Q. Chen, Q. Xia, C. He, C. Kang, "Incentive compatible pool-based electricity market design and implementation: A Bayesian mechanism design approach," Applied Energy, vol. 158, pp. 508-518, 2015.

[13] J. Aghaei, M. I. Alizadeh, "Demand response in smart electricity grids equipped with renewable energy sources: A review," Renewable and Sustainable Energy Reviews, vol. 18, pp. 64-72, 2013.

[14] V. Rious, Y. Perez, F. Roques, "Which electricity market design to encourage the development of demand response?," Economic Analysis and Policy, vol. 48, pp. 128-138, 2015.

[15] A. Anees, Y. P. Chen, "True real time pricing and combined power scheduling of electric appliances in residential energy management system," Applied Energy, vol. 165, pp. 592-600, 2016.

[16] S. Sekizaki, I. Nishizaki, T. Hayashida, "Electricity retail market model with flexible price settings and elastic price-based demand responses by consumers in distribution network," Electrical Power and Energy Systems, vol. 81, pp. 371-386, 2016.

[17] M. Mazidi, H. Monsef, P. Siano, "Incorporating price-responsive customers in day-ahead scheduling of smart distribution networks," Energy Conversion and Management, vol. 115, pp. 103-116, 2016.

[18] M. Doostizadeh, H. Ghasemi, "A day-ahead electricity pricing model based on smart metering and demand-side management," Energy, vol. 46, no. 1, pp. 221-230, 2012.

[19] Y. F. B. Jiang, "Dynamic Residential Demand Response and Distributed Generation Management in Smart Microgrid with Hierarchical Agents," Energy Procedia, vol. 12, pp. 76-90, 2011.

[20] M. Cepeda, M. Saguan, "Assessing long-term effects of demand response policies in wholesale electricity markets," Electrical Power and Energy Systems, vol. 74, pp. 142-152, 2016.

[21] M. Shafie-khah, E. Heydarian-Forushani, G. J. Osório, F. A. S. Gil, J. Aghaei, M. Barani, J. P. S. Catalão, "Optimal Behavior of Electric Vehicle Parking Lots as Demand Response Aggregation Agents," IEEE Transactions on Smart Grid, vol. PP, no. 99, pp. 1-12, 2015.

[22] M. Shafie-khah, E. Heydarian-Forushani, M. E. H. Golshan, P. Siano, M. P. Moghaddam, M. K. Sheikh-El-Eslami, J. P. S. Catalão, "Optimal trading of plug-in electric vehicle aggregation agents in a market environment for sustainability," Applied Energy, vol. 162, pp. 601-612, 2016.

[23] N. Mahmoudi, T. K. Saha, M. Eghbal, "A new trading framework for demand response aggregators," in 2014, National Harbor, MD, 2014 IEEE PES General Meeting, Conference \& Exposition.

[24] A. G. Zamani, A. Zakariazadeh, S. Jadid, "Day-ahead resource scheduling of a renewable energy based virtual power plant," Applied Energy, vol. 169, pp. 324-340, 2016.

[25] J. S. Vardakas, N. Zorba, C. V. Verikoukis, "Performance evaluation of power demand scheduling scenarios in a smart grid environment," Applied Energy, vol. 142, pp. 164-178, 2015.

[26] M. Alipour, B. Mohammadi-Ivatloo, K. Zare, "Stochastic risk-constrained short-term scheduling of industrial cogeneration systems in the presence of demand response programs," Applied Energy, vol. 136, pp. 393-404, 2014.

[27] B. D. H. Kiran, M. S. Kumari, "Demand response and pumped hydro storage scheduling for balancing wind power 
uncertainties: A probabilistic unit commitment approach," Electrical Power and Energy Systems, vol. 81, pp. 114-122, 2016.

[28] L. Ju, Z. Tan, J. Yuan, Q. Tan, H. Li, F. Dong, "A bi-level stochastic scheduling optimization model for a virtual power plant connected to a wind-photovoltaic-energy storage system considering the uncertainty and demand response," Applied Energy, vol. 171, p. 184-199, 2016.

[29] A. Zakariazadeh, S. Jadid, P. Siano, "Stochastic operational scheduling of smart distribution system considering wind generation and demand response programs," Electrical Power and Energy Systems, vol. 63, pp. 218-225, 2014.

[30] H. G. Kwag, J. O. Kim, "Optimal combined scheduling of generation and demand response with demand resource constraints," Applied Energy, vol. 96, pp. 161-170, 2012.

[31] H. Falsafi, A. Zakariazadeh, S. Jadid, "The role of demand response in single and multi-objective wind-thermal generation scheduling: A stochastic programming," Energy, vol. 64, pp. 853-867, 2014.

[32] E. Heydarian-Forushani, M. E. H. Golshan, M. P. Moghaddam, M. Shafie-khah, J. P. S. Catalão, "Robust scheduling of variable wind generation by coordination of bulk energy storages and demand response," Energy Conversion and Management, vol. 106, pp. 941-950, 2015.

[33] A. Samimi, A. Kazemi, P. Siano, "Economic-environmental active and reactive power scheduling of modern distribution systems in presence of wind generations: A distribution market-based approach," Energy Conversion and Management, vol. 106, pp. 495-509, 2015.

[34] P. Faria, Z. Vale, J. Baptista, "Constrained consumption shifting management in the distributed energy resources scheduling considering demand response," Energy Conversion and Management, vol. 93, pp. 309-320, 2015.

[35] R. Wang, P. Wang, G. Xiao, "A robust optimization approach for energy generation scheduling in microgrids," Energy Conversion and Management, vol. 106, pp. 597-607, 2015.

[36] N. I. Nwulu, X. Xia, "Multi-objective dynamic economic emission dispatch of electric power generation integrated with game theory based demand response programs," Energy Conversion and Management, vol. 89, pp. 963-974, 2015.

[37] G. R. Aghajani, H. A. Shayanfar, H. Shayeghi, "Presenting a multi-objective generation scheduling model for pricing demand response rate in micro-grid energy management," Energy Conversion and Management, vol. 106, pp. 308-321, 2015.

[38] C. Á. Bel, M. A. Ortega, G. E. Escrivá, A. G. Marín, "Technical and economical tools to assess customer demand response in the commercial sector," Energy Conversion and Management, vol. 50, pp. 2605-2612, 2009.

[39] C. Yan, X. Xue, S. Wang, B. Cui, "A novel air-conditioning system for proactive power demand response to smart grid," Energy Conversion and Management, vol. 102, pp. 239-246, 2015.

[40] H. Shayeghi, B. Sobhani, "Integrated offering strategy for profit enhancement of distributed resources and demand response in microgrids considering system uncertainties," Energy Conversion and Management, vol. 87, pp. 765-777, 2014.

[41] H. Morais, T. Sousa, J. Soares, P. Faria, Z. Vale, "Distributed energy resources management using plug-in hybrid electric vehicles as a fuel-shifting demand response resource," Energy Conversion and Management, vol. 97, pp. 78-93, 2015.

[42] B. Cui, S. Wang, C. Yan, X. Xue, "Evaluation of a fast power demand response strategy using active and passive building cold storages for smart grid applications q," Energy Conversion and Management, vol. 102, pp. 227-238, 2015.

[43] S. Nojavan, H. A. Aalami, "Stochastic energy procurement of large electricity consumer considering photovoltaic, windturbine, micro-turbines, energy storage system in the presence of demand response program," Energy Conversion and Management, vol. 103, pp. 1008-1018, 2015.

[44] Z. Tan, L. Ju, B. Reed, R. Rao, D. Peng, H. Li, G. Pan, "The optimization model for multi-type customers assisting wind power consumptive considering uncertainty and demand response based on robust stochastic theory," Energy Conversion and Management, vol. 105, pp. 1070-1081, 2015.

[45] M. Alcázar-Ortega, C. Calpe, T. Theisen, J. F. Carbonell-Carretero, "Methodology for the identification, evaluation and prioritization of market handicaps which prevent the implementation of Demand Response: Application to European electricity markets," Energy Policy, vol. 86, pp. 529-543, 2015.

[46] T. S. Genc, "Measuring demand responses to wholesale electricity prices using market power indices," Energy Economics, vol. 56, pp. 247-260, 2016.

[47] M. Tanaka, Y. Chen, "Market power in emissions trading: Strategically manipulating permit price through fringe firms," Applied Energy, vol. 96, pp. 203-211, 2012.

[48] C. A. Díaz, F. A. Campos, J. Villar, "Existence and uniqueness of conjectured supply function equilibria," Electrical Power and Energy Systems, vol. 58, pp. 266-273, 2014.

[49] M. Dolmatova, A. Vasin, H. Gao, "Supply function auction for linear asymmetric oligopoly: equilibrium and convergence," Procedia Computer Science, vol. 55, pp. 112 -118, 2015. 
[50] C. A. Díaz, J. Villar, F. A. Campos, M. Á. Rodríguez, "A new algorithm to compute conjectured supply function equilibrium in electricity markets," Electric Power Systems Research, vol. 81, pp. 384-392, 2011.

[51] S. Soleymani, "Nash equilibrium strategies of generating companies (Gencos) in the simultaneous operation of active and reactive power market, with considering voltage stability margin," Energy Conversion and Management, vol. 65, pp. 292298, 2013.

[52] P. Holmberg, "Numerical calculation of an asymmetric supply function equilibrium with capacity constraints," European Journal of Operational Research, vol. 199, pp. 285-295, 2009.

[53] P. Holmberg, D. Newbery, "The supply function equilibrium and its policy implications for wholesale electricity auctions," Utilities Policy, vol. 18, pp. 209-226, 2010.

[54] T. Niknam,S, Sharifinia, R. Azizipanah-Abarghooee, "A new enhanced bat-inspired algorithm for finding linear supply function equilibrium of GENCOs in the competitive electricity market," Energy Conversion and Management, vol. 76, pp. 1015-1028, 2013.

[55] N. Rezaei, M. Kalantar, "Hierarchical energy and frequency security pricing in a smart microgrid: An equilibrium-inspired epsilon constraint based multi-objective decision making approach," Energy Conversion and Management, vol. 98, pp. 533$543,2015$.

[56] G. Ghatikar, S. Mashayekh, M. Stadler, R. Yin, Z. Liu, "Distributed energy systems integration and demand optimization for autonomous operations and electric grid transactions," Applied Energy, vol. 167, pp. 432-448, 2016.

[57] D. Olsen, et. al., "Grid Integration of Aggregated Demand Response, Part 1: Load Availability Profiles and Constraints for the Western Interconnection," 2013. [Online]. Available: http://drrc.lbl.gov/publications/grid-integration-aggregateddemand.

[58] J. L. Mathieu, M. G. Vaya, G. Andersson, "Uncertainty in the Flexibility of Aggregations of Demand Response Resources," in Industrial Electronics Society, IECON 2013 - 39th Annual Conference of the IEEE.

[59] D. Kirschen, G. Strbac, Fundamentals of Power System Economics, John Wiley \& Sons, Ltd, 2004.

[60] R. D. Zimmerman, C. E. Murillo-Sánchez, and R. J. Thomas, "MATPOWER: Steady-State Operations, Planning and Analysis Tools for Power Systems Research and Education," IEEE Trans. Power Syst. , vol. 26, no. 1, pp. 12-19, Feb 2011.

[61] M. Baran, F. F. Wu, "Optimal sizing of capacitors placed on a radial distribution system," IEEE Transactions on Power Delivery, vol. 4, no. 1, pp. 735-743, 1989.

[62] R. Baldick, R. Grant and E. Kahn, "Theory and application of linear supply function equilibrium in electricity markets," Journal of Regulatory Economics, vol. 25, no. 2, pp. 143-167, 2004.

[63] F. Boneti and M. E. Zuanon, "Equilibrium a la Cournot and Supply Function," Journal of Regulatory Economics Equilibrium in Electricity Markets under Linear Price/Demand and Quadratic Costs, vol. 7, no. 40, pp. 1993-1998, 2012.

[64] H. M. Soliman, A. Leon-Garcia, "Game-Theoretic Demand-Side Management With Storage Devicesforthe Future Smart Grid," IEEE Trans. Smart Grids, vol. 5, no. 3, pp. 1475-1485, 2014.

[65] A. Belov, A. Vasenev, P. J. M. Havinga, N. Meratnia, B. J. van der Zwaag, "Reducing user discomfort in direct load control of domestic water heaters," in Smart Grid Technologies - Asia (ISGT ASIA), IEEE Innovative, Bangkok, 2015.

[66] "Powersim Simulation PSIM," [Online]. Available: https://powersimtech.com/products/psim/.

[67] M. Thornton, H. Smidt, V. Schwarzer, R. Ghorbani, "Internet-of-Things Hardware-in-the-Loop Simulation Testbed," in Hawaii international conference on system sciences (HICSS) , [in preparation]. 